\title{
OPEN Identification of missed viruses by metagenomic sequencing of clinical respiratory samples from Kenya
}

\author{
My V. T. Phan ${ }^{1,2 \varpi}$, Charles N. Agoti ${ }^{3,4}$, Patrick K. Munywoki $i^{3,4}$, Grieven P. Otieno ${ }^{3}$, \\ Mwanajuma Ngama ${ }^{3}$, Paul Kellam ${ }^{1,5}$, Matthew Cotten $^{1,2,6}$ \& D. James Nokes ${ }^{3,7 凶}$
}

Pneumonia remains a major cause of mortality and morbidity. Most molecular diagnoses of viruses rely on polymerase chain reaction (PCR) assays that however can fail due to primer mismatch. We investigated the performance of routine virus diagnostics in Kilifi, Kenya, using random-primed viral next generation sequencing (viral NGS) on respiratory samples which tested negative for the common viral respiratory pathogens by a local standard diagnostic panel. Among 95 hospitalised pneumonia patients and 95 household-cohort individuals, analysis of viral NGS identified at least one respiratory-associated virus in 35 (37\%) and 23 (24\%) samples, respectively. The majority (66\%; 42/64) belonged to the Picornaviridae family. The NGS data analysis identified a number of viruses that were missed by the diagnostic panel (rhinovirus, human metapneumovirus, respiratory syncytial virus and parainfluenza virus), and these failures could be attributed to PCR primer/probe binding site mismatches. Unexpected viruses identified included parvovirus B19, enterovirus D68, coxsackievirus A16 and A24 and rubella virus. The regular application of such viral NGS could help evaluate assay performance, identify molecular causes of missed diagnoses and reveal gaps in the respiratory virus set used for local screening assays. The results can provide actionable information to improve the local pneumonia diagnostics and reveal locally important viral pathogens.

Pneumonia is a leading cause of illness globally ${ }^{1}$ and determining the aetiologies of the disease would help case management, especially with regard to bacterial versus viral infections and decisions to use antibiotics as firstline therapeutic treatment. Polymerase chain reaction (PCR) methods are frequently used for viral diagnosis but costs and logistics limit the number of pathogens included in diagnostic panels. Additionally, mismatches in primers can cause missed diagnoses with evolving RNA pathogens ${ }^{2,3}$. Other viruses which are not included in the diagnostic panels due to costs may also contribute to missed diagnoses. Studies in Kilifi (coastal Kenya) to investigate viral aetiologies of pneumonia in paediatric patients and in the community identified respiratory viruses with these methods ${ }^{4-9}$; however, a proportion of samples collected from symptomatic children were negative for any respiratory viruses in the diagnostic screening panel.

We evaluated the ability of next-generation sequencing (NGS) to identify viruses missed by the diagnostic panel by evaluating samples from patients with respiratory disease which tested negative for common respiratory viral pathogens. A random priming (agnostic) NGS method was employed directly on clinical samples to detect viruses present in the samples by viral metagenomics ${ }^{10,11}$ without prior knowledge or specific PCR primers. The data generated from agnostic viral metagenomics of clinical samples were used to control the quality of the diagnostics (e.g. determine the presence of unanticipated viruses and variants of unexpected viruses on diagnostic panel), in order to help define the sensitivity of the diagnostic panels, reveal additional common respiratory pathogens, and improve future diagnostic assays for the region. These data will also help infectious

\footnotetext{
${ }^{1}$ Virus Genomics, Wellcome Trust Sanger Institute, Hinxton, UK. ${ }^{2}$ MRC/UVRI \& LSHTM Uganda Research Unit, Entebbe, Uganda. ${ }^{3}$ Epidemiology and Demography Department, KEMRI -Wellcome Trust Research Programme, Kilifi, Kenya. ${ }^{4}$ School of Health and Human Sciences, Pwani University, Kilifi, Kenya. ${ }^{5}$ Division of Infectious Diseases, Department of Medicine, Imperial College London, London, UK. ${ }^{6}$ MRC-University of Glasgow Centre for Virus Research, Glasgow, UK. ${ }^{7}$ School of Life Sciences and Zeeman Institute for Systems Biology and Infectious Disease Epidemiology Research (SBIDER), University of Warwick, Coventry, UK. 『email: my.phan@lshtm.ac.uk; JNokes@kemri-wellcome.org
} 


\begin{tabular}{|l|l|l|l|l|}
\hline Virus species & Virus family & KCH cohort & Household cohort & Grounds for diagnostic failure \\
\hline HRV-A & Picornaviridae & 4 & 4 & Primer/probe mismatch \\
\hline HRV-B & Picornaviridae & 3 & 4 & Primer/probe mismatch \\
\hline HRV-C & Picornaviridae & 11 & 2 & Primer/probe mismatch \\
\hline HMPV & Pneumoviridae & 4 & 2 & Primer/probe mismatch \\
\hline RSVB & Pneumoviridae & 3 & 0 & Primer/probe mismatch \\
\hline HPIV-1 & Paramyxoviridae & 0 & 1 & Primer/probe mismatch \\
\hline DENV-2 & Flaviviridae & 1 & 0 & Not in panel \\
\hline HHV-5 & Herpesviridae & 2 & 0 & Not in panel \\
\hline HSV-1 & Herpesviridae & 2 & 0 & Not in panel \\
\hline Bocavirus & Parvoviridae & 1 & 3 & Not in panel \\
\hline Parvovirus B19 & Parvoviridae & 1 & 0 & Not in panel \\
\hline Echovirus E1 & Picornaviridae & 0 & 1 & Not in panel \\
\hline EV-D68 & Picornaviridae & 4 & 0 & Not in panel \\
\hline CV-A16 & Picornaviridae & 1 & 0 & Not in panel \\
\hline CV-A24 & Picornaviridae & 0 & 6 & Not in panel \\
\hline Parechovirus & Picornaviridae & 0 & 1 & Not in panel \\
\hline Poliovirus & Picornaviridae & 1 & 0 & Not in panel \\
\hline Rubella & Matonaviridae & 2 & 0 & Not in panel \\
\hline Total virus identified & & 24 & \\
\hline
\end{tabular}

Table 1. Detected respiratory viruses, sample source and our explanation for diagnostic failure.

disease clinicians to suspect potential causes that are not routinely included in the diagnosis if the clinical presentations warrant such a diagnosis.

\section{Results}

The detection of viruses. As shown in Table 1, viral NGS identified at least one syndrome-associated mammalian virus in 35 of 95 Kilifi County Hospital (KCH) inpatient samples (36.8\%) and 23 of 95 household cohort (study investigating Who-Acquires-Infection-From-Whom, WAIFW) samples (24.2\%), leading to an overall 30.5\% "missed virus detection rate". Among 58 samples that yielded a virus were six samples with mixed virus infections ( 5 in $\mathrm{KCH}$ patients and 1 in the household cohort). In the five $\mathrm{KCH}$ mixed infections, four were human rhinovirus A (HRV-A)/respiratory syncytial virus B (RSVB), HRV-A/Enterovirus D68 (EV-D68), Bocavirus/human rhinovirus B (HRV-B), or EV-D68/Coxsackievirus A16 (CV-A16) and one sample showed two distinct human rhinovirus C (HRV-C) strains. Overall, 64 viruses were identified; these were either missed viruses (viruses included in the standard diagnostic panel but the assay was negative; $n=38$ ) or unexpected viruses (viruses not part of the standard diagnostic panel; $n=26$ ).

Missed viruses. The number of respiratory viruses (with contigs $\geq 1000 \mathrm{nt}$ ) that had been missed by the standard diagnostic panel included 28 human rhinovirus (HRV) in 27 samples (14.2\%; 27/190), one human parainfluenza virus 1 (HPIV-1) $(0.5 \% ; 1 / 190)$, three RSVB $(1.6 \% ; 3 / 190)$, and six human metapneumovirus (HMPV) $(3.2 \%$; 6/190) (Table 1). For these missed viruses, nucleotide mismatch between the diagnostic primers and probes and the viral target sequence were identified, potentially accounting for detection failures (see below).

Analysis of primer mismatches. Likely causes of missed diagnoses are mismatches between the primer/ probes and viral target sites. For all viral contigs $\geq 1000 \mathrm{nt}$, if the virus family was part of the diagnostic panel, target sites were examined for differences from the primer/probe. For HRV-A, HRV-B, HRC-V, HMPV and HPIV-1, a number of nucleotide changes were observed in target sites and most were consistent with failed or suboptimal diagnostic tests (Figs. 1, 2). For the RSVB genomes detected, there were nucleotide changes in the probe targets; an updated panel of primers/probe was recently developed and used successfully ${ }^{1}$.

Unexpected viruses. An advantage of the agnostic viral NGS is the ability to detect viruses present in a specimen without a prior knowledge of virus genome sequence for primer design. In the 190 samples, 26 unexpected viruses from five families of viruses were identified, none were included in the standard diagnostic panel (Table 1). Most unexpected viruses (50\%; 13/26) were Picornaviridae, genus Enterovirus, species A (CV-A16, $\mathrm{n}=1$ ), species B (Echovirus E1, $\mathrm{n}=1)$, species C (CV-A24, $\mathrm{n}=6$ and human poliovirus 2 strain Sabin, $n=1)$, and species D (EV-D68, n=4). The Parvoviridae Human bocavirus (HBoV) and parvovirus B19 (B19) were identified in four and one sample (Table 1). Rubella virus (RVi) was detected in two $\mathrm{KCH}$ paediatric patients with very different clinical presentations (further details below). 
A - Primer check HMPV-A

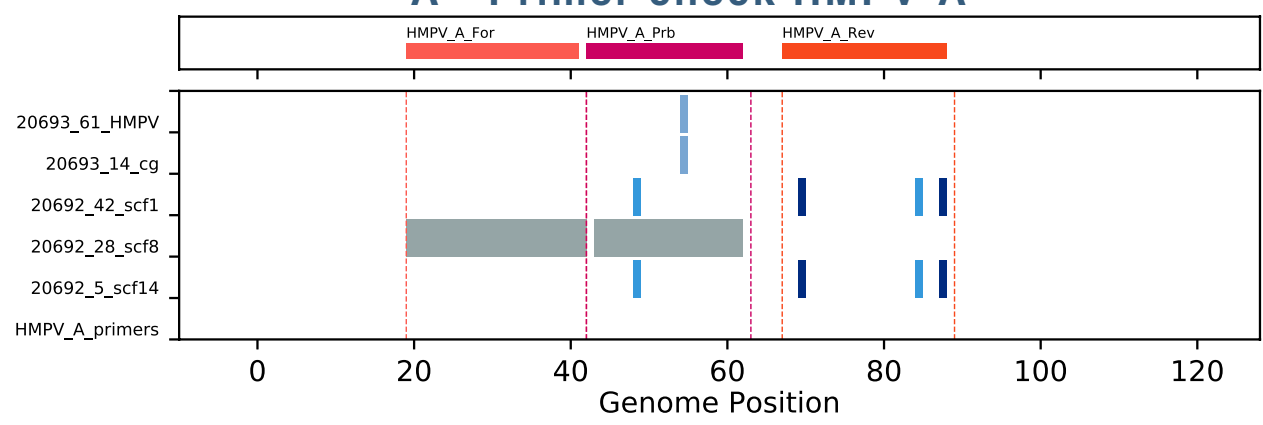

B - Primer check HMPV-B

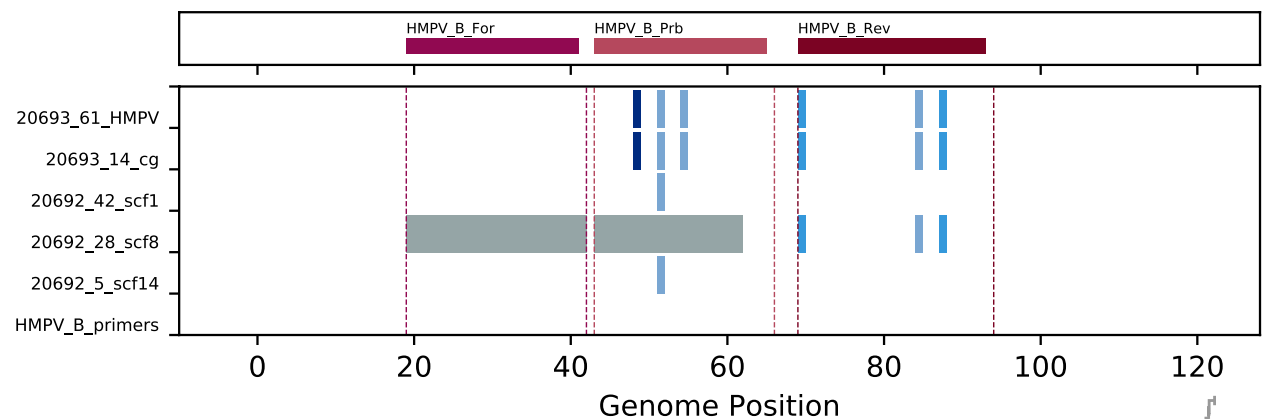

Genome Position

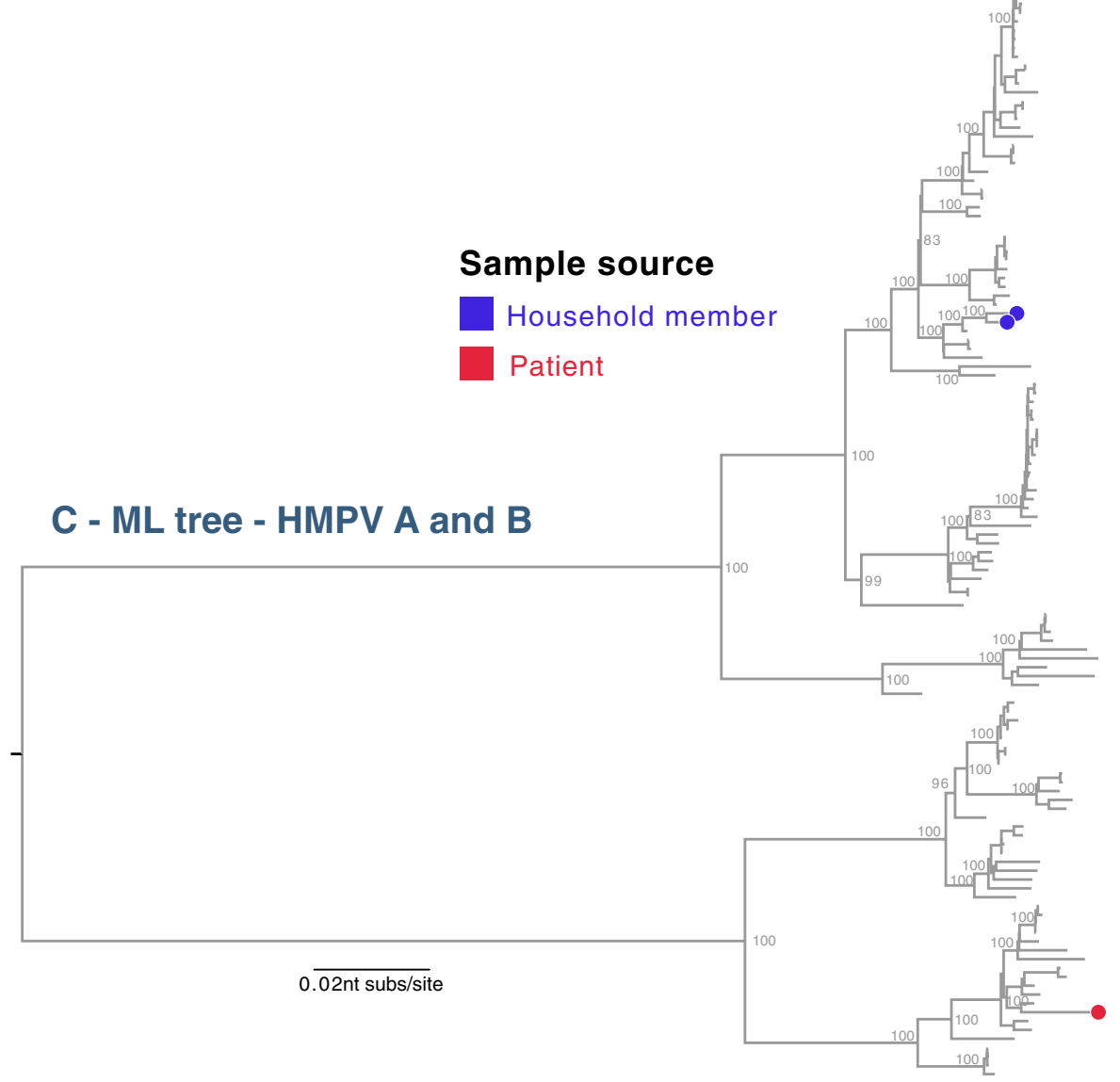

Figure 1. Human metapneumovirus (HMPV) identified in the study. (A) The diagnostic primers and probe target sites in the Kilif HMPV genotype A genomes and contigs were examined. All viral contigs from each virus family or type were aligned using MAFFT ${ }^{25}$, and the alignment was trimmed to a 100-200 nt region surrounding the primer and probe target sites. Nucleotide differences between the expected primer and probe target sites and the actual contig sequences were identified and plotted in shades of blue and gaps in contig sequences were indicated in grey. (B) As in (A) but for HMPV genotype B. (C) Maximum-likelihood (ML) phylogenetic tree of HMPV genomes. Local strains on the phylogenetic tree were indicated by circles coloured in blue indicating household member and in red indicating $\mathrm{KCH}$ patients. The tree was mid-point rooted for clarity and horizontal branch lengths were drawn to the scale of nucleotide substitutions per site. The tree comparing local HMPV genomes to global genomes suggested that the local HMPV belonged to genotype A2 and B1. 
HRV-A

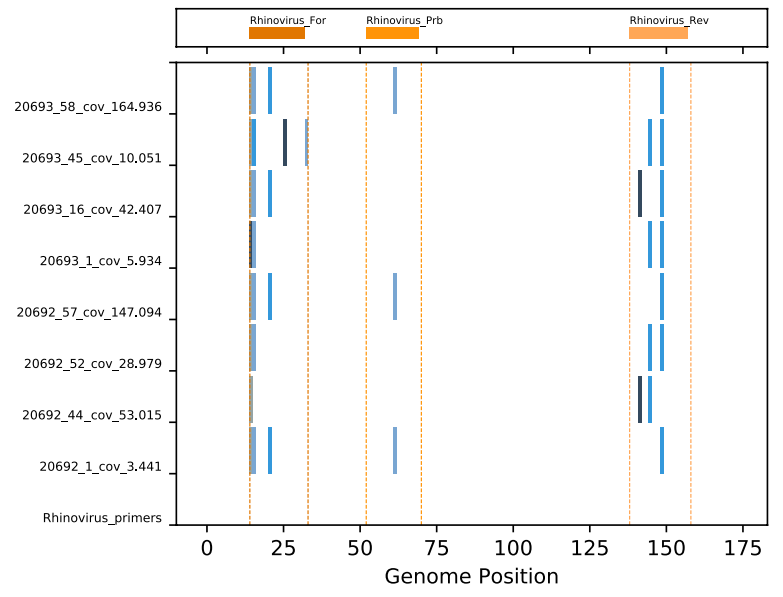

HRV-B

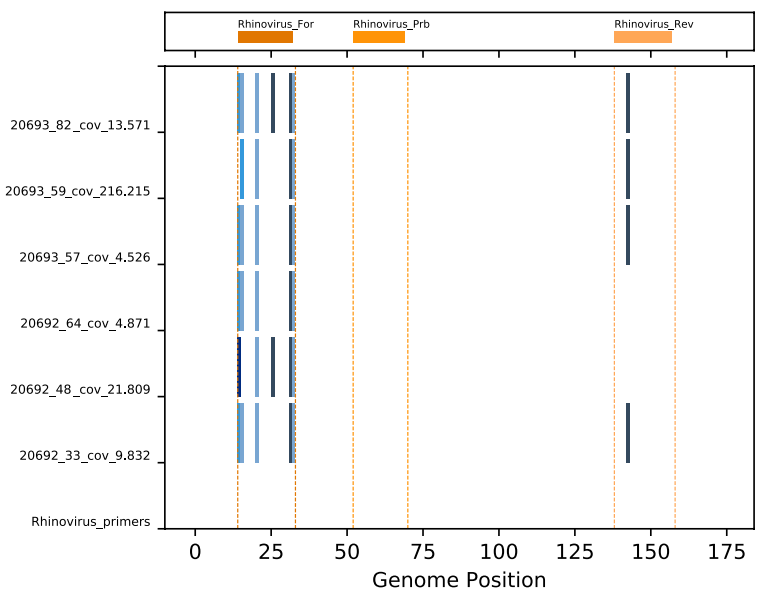

HRV-C

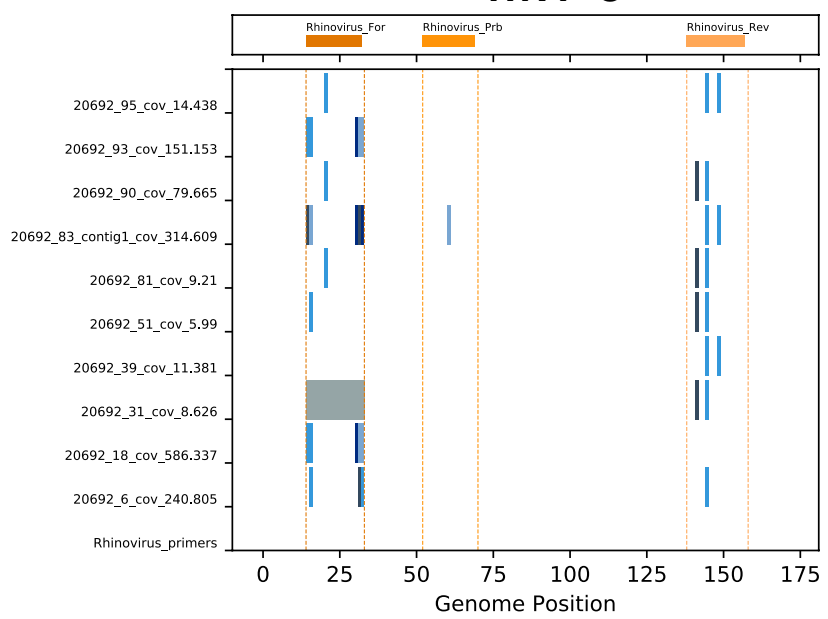

HPIV-1

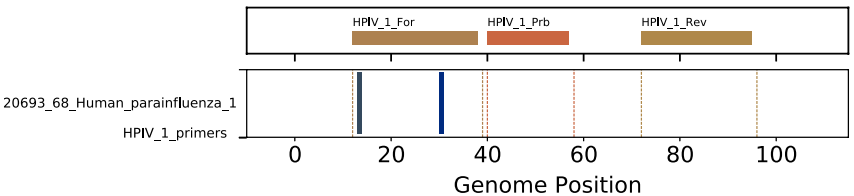

Figure 2. Diagnostic primers and probes check for human rhinoviruses (HRV) and human parainfluenza virus identified from the study. The diagnostic primers and probes target sites in the Kilifi HRV-A, HRV-B, HRV-C and HPIV-1 genomes were examined (see Fig. 1 legend for detailed methods). For each contig, nucleotide changes from the expected target sites were indicated by vertical blue lines, gaps in the sequence were indicated by grey bars.

Human metapneumovirus. Human metapneumovirus (HMPV) infection is frequent in young children ${ }^{12}$ and was identified in six samples (Table 1). The diagnostic primer targets in the genomes showed mismatches (Fig. 1, panels A, B) that could explain the missed HMPV diagnostics. A maximum-likelihood phylogenetic tree comparing the HMPV complete genomes from the local strains to global circulating strains showed the two HMPV from the household cohort were genotype A2; the HMPV from the KCH patient was genotype B1, closest to strains KC562240 (A2) and KF530179 (B1) from Australia in 2003 (Fig. 1, panel C). The reported HMPV sequences were also compared with local Kenya HMPV short sequences available from GenBank, all fell into similar lineages (Supplementary Fig. S1, panel B) and were likely missed because of primer mismatch rather than because they were a new lineage.

Enterovirus genus. Among 64 viruses detected, the majority were from the Enterovirus genus, Picornaviridae family $(\mathrm{N}=42 ; 66 \%)$. Apart from HRV $(\mathrm{N}=28)$, viruses from the Enterovirus species were not included in the routine screening.

Rhinovirus species, Enterovirus genus. The most abundant Enteroviruses identified were Rhinovirus species A, B and C, with 28 complete or partial genomes identified. All three sets of diagnostic primers used at the time showed multiple mismatches with the genome target sites that could account for the 28 missed HRV cases (Fig. 2). A high diversity of circulating HRV has been noted in this region ${ }^{6,13}$ as shown in phylogenetic trees comparing local HRV identified from this study with global HRV genomes (Supplementary Fig. S2).

Enterovirus A species (CV-A16), Enterovirus genus. Coxsackievirus A16 (CV-A16), enterovirus 71 (EV-71) and several additional Enterovirus species are associated with hand, foot and mouth disease (HFMD $)^{14}$. The 22-month old patient infected with CV-A16 was hospitalized at $\mathrm{KCH}$ with pneumonia, but presented no 

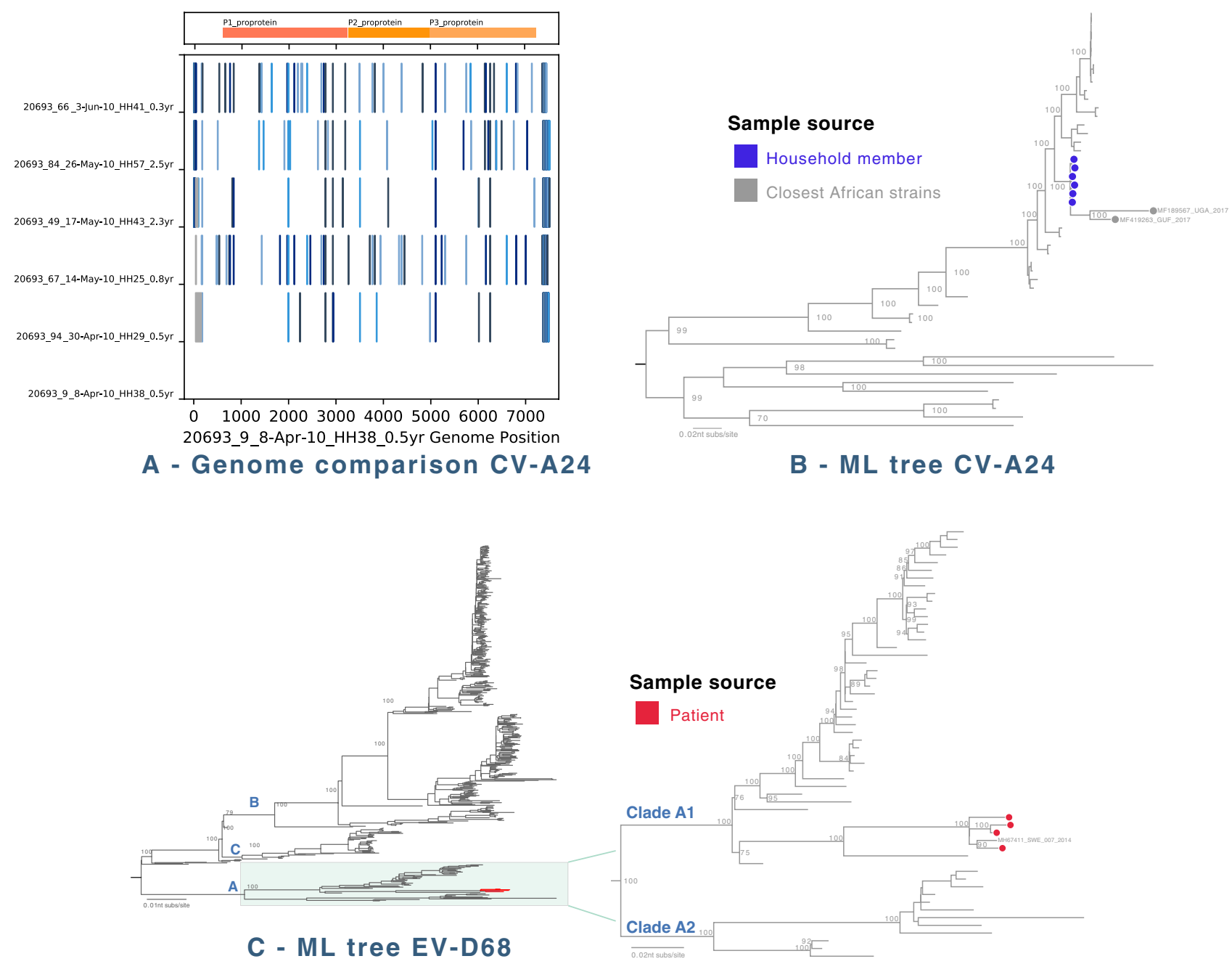

Figure 3. (A) Genome comparison of identified human coxsackievirus A24 (CV-A24) from the study. Kilifi CV-A24 genomes were examined and compared against the earliest Kilifi CV-A24 genome identified (20693_9, sample collected on 8 April 2010). For each genome, nucleotide changes from the first CV-A24 genome (20693_3) were indicated by vertical blue lines, gaps in the sequence were indicated by grey bars. (B) Maximumlikelihood phylogenetic tree of CV-A24 genomes. The ML tree compared 6 Kilifi CV-A24 genomes (all from household cohort, indicated as blue circles) to global genomes. The tree was mid-point rooted for clarity and horizontal branch lengths were drawn to the scale of nucleotide substitutions per site, and significant bootstrap values were shown for major nodes. (C) Human enterovirus D68 (EV-D68) maximum-likelihood phylogenetic tree. Maximum-likelihood phylogenetic tree were inferred comparing 4 Kilifi EV-D68 genomes (all from $\mathrm{KCH}$ pneumonia patients cohort, highlighted in red) to global genomes. The tree was mid-point rooted for clarity and horizontal branch lengths were drawn to the scale of nucleotide substitutions per site, and significant bootstrap values were shown for major nodes. These four local EV-D68 viruses belonged to clade A1, as shown in the zoomed out tree.

clinical HFMD symptoms, and was discharged home after 3 days. Phylogenetically, the patient's CV-A16 virus genome was closely related to a CV-A16 strain identified from an Ethiopian child in April 2016 (Supplementary Fig. S1, panel A) ${ }^{15}$.

Enterovirus C species (CV-A24), Enterovirus genus. Coxsackievirus A24 (CV-A24) was identified in six samples, all from a 2-month period (8 April thorugh 3 June 2010) in the household study (Fig. 5). The six infected individuals were aged 8.5 to 33 months, and came from different households. None of these children presented with conjunctivitis, one had diarrhea and all had rhinorrhea. The identified CV-A24 genomes showed 12 to $146 \mathrm{nt}$ differences and very few shared SNPs (Fig. 3, panel A), suggesting that the viruses were not directly transmitted between the 6 individuals. The samples were selected to cover as many households as possible over the entire cohort time period, thus the observed diversity may reflect a much larger outbreak that would account for the number of nucleotide changes. This is also consistent with the monophyletic phylogeny for the six genomes (Fig. 3, panel B). When analyzed with all available CV-A24 genomes from GenBank, the local CV-A24 sequences formed a monophyletic group closest to sequences from Uganda (GenBank MF189567) 


\begin{tabular}{|l|l|l|}
\hline Features & Case A & Case B \\
\hline Gender & Female & Female \\
\hline Place of birth & Home & Home \\
\hline Age at admission & 10 days & 27 days \\
\hline Discharge & 2 days after admission & 4 days after admission \\
\hline Discharge type & Death & Alive \\
\hline Discharge diagnosis & Neonatal sepsis and haemorrhage & Neonatal sepsis \\
\hline Conscious level & Lethargic & Normal \\
\hline Arrest type & Respiratory & \\
\hline Arrest CPR & Bag and mask & \\
\hline HDU admission & Yes & No \\
\hline Additional comments & Meningitis with congenital cataracts & \\
\hline MMR vaccination & No & No \\
\hline
\end{tabular}

Table 2. Infection features of two rubella cases. $C P R$ cardiopulmonary resuscitation, $H D U$ high dependency unit.

and French Guiana (GenBank MF419263) which were associated with ocular inflammation or acute haemorrhagic conjunctivitis (AHC) in $2017^{16}$ (Fig. 3, panel B).

Enterovirus C species (human poliovirus), Enterovirus genus. The detection of human poliovirus type 2 was likely due to viral shedding after oral poliovirus vaccination. The child in whom this isolate was detected was 6 weeks old at the time of sampling, and had received a dose of oral polio vaccine (OPV) 4 days prior. The child was hospitalised with cough and difficulty breathing, and was discharged home after 3 days. The human poliovirus 2 genome obtained was identical to the vaccine strain Sabin 2 (GenBank AY184220). Health authorities were informed about this finding.

Enterovirus D species (EV-D68), Enterovirus genus. Four EV-D68 were identified in the hospitalised cohort over a 5-month period (8 April through 318 July 2010). Two of these patients were co-infected with additional Enterovirus strains, CV-A16 or HRV-A. The four children with EV-D68 were hospitalised with severe pneumonia for 3-6 days, and were discharged with no report of neurological symptom.

Phylogenetic analysis (Fig. 3, panel C) suggested that the four Kilifi EV-D68 viruses were clade A1 and closely related to a strain identified from a respiratory patient in 2014 in Sweden (GenBank MH674114) and to a Canadian EV-D68 strain in 2014 (GenBank KP455258).

Matonaviridae family, Rubivirus genus (Rubella virus). Rubella is a contagious typically mild disease caused by rubella virus (RVi), a single-stranded RNA virus in the Matonaviridae family, Rubivirus genus, infecting people of any age. However, primary RVi infection during the first trimester of pregnancy may result in congenital rubella syndrome (CRS) or miscarriage. Common sequelae of CRS include deafness, glaucoma and retinopathy and heart defects. Rubella infections can be prevented by highly effective rubella vaccine. In Kenya, national rubella vaccination was not implemented until October 2016 and there was no surveillance of RVi prevalence or CRS incidence ${ }^{17}$.

$\mathrm{RVi}$ was detected in two KCH patients through this NGS study (Table 2). They were 10 days and 27 days old at the time of hospitalisation, and had admission diagnoses of neonatal sepsis. Their hospital admissions occurred 5 weeks apart. Phylogenetic analysis on the complete RVi genomes compared with all available RVi genomes available from Genbank indicated that the two Kilifi RVi genomes were similar (60nt differences, 99.4\% identity) and belonged to the same genotype (genotype 2B, Fig. 4). Rubella is not routinely screened for or suspected in respiratory infections or neonatal sepsis. Identification of RVi in two neonatal patients in the context of absent or low vaccination coverage in LMIC settings, would alert clinicians to consider this virus in their diagnoses.

Parvoviridae family. We identified parvovirus B19 (B19) in a 5-month old hospitalised patient with very severe pneumonia and anaemia. Tests for malaria were negative, and the patient was discharged after 3 days. The identified B19 virus genome belonged to genotype 1A, similar to other global B19 sequences as shown in the phylogenetic tree comparing the local B19 genome to global sequences (genotype 1A, Supplementary Fig. S1, panel C).

Human bocavirus (HBoV) type 1 was identified in a hospitalised child with malnutrition, severe pneumonia and diarrhoea, and in three children with upper respiratory infections from different households. These $4 \mathrm{HBoV} 1$ genomes clustered in 2 sub-lineages within genotype 1 when compared with all global sequences as shown in Supplementary Fig. S1, panel D.

Other viruses. Viruses detected at low frequency included HPIV-1 (one case), human parechovirus (one case), human herpesvirus 5 (HHV-5; two cases), human herpes simplex virus (HSV-1; one case), Dengue Virus type 2 (DENV-2; one case) and echovirus E1 (one case). 


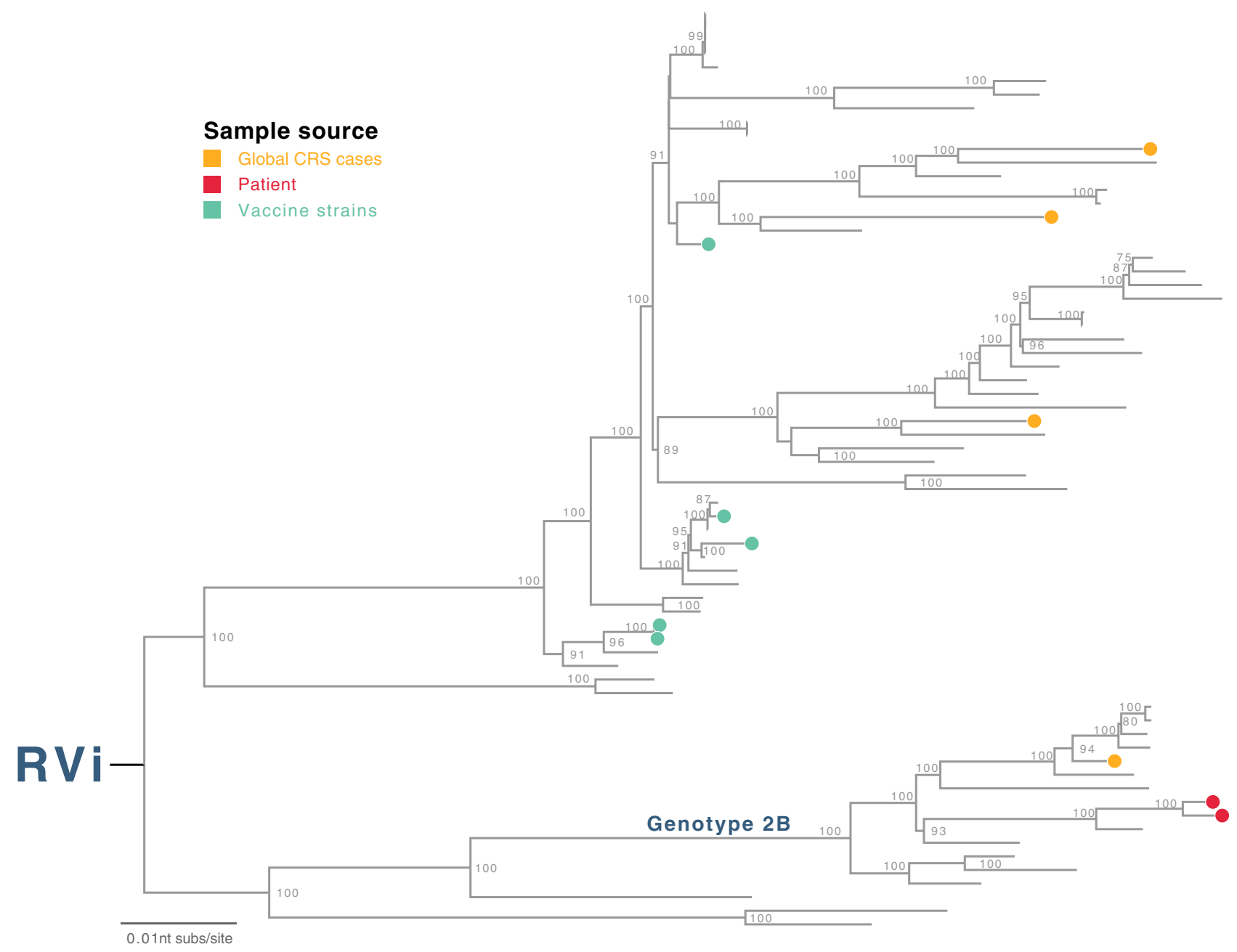

Figure 4. Human rubella virus (RVi). Maximum-likelihood phylogenetic tree was inferred comparing two RVi genomes (all from $\mathrm{KCH}$ pneumonia patients cohort, indicated as red circles) to global genomes. The global RVi strains identified in confirmed CRS cases were indicated as orange circles in the tree, and RVi vaccine strains were indicated as green circles. The tree was mid-point rooted for clarity and horizontal branch lengths were drawn to the scale of nucleotide substitutions per site, and significant bootstrap values were shown for major nodes.

Detection timeline. The date of collection of specimens that were test negative using the routine viral panel assay, and their NGS viral detection results, are plotted by time (Fig. 5). The various HMPV, HRV-A, HRV-B and HRV-C positive samples are distributed throughout the observation period and occurred in both study groups (KCH and WIAFW). CV-A24 and EV-D68 positive samples were detected over discrete time periods (2 months in 2010 and 5 months in 2015, respectively) as mentioned above. The other observed viruses were too few to make strong conclusions about their temporal distribution.

\section{Discussion}

Respiratory infections are a leading cause of morbidity and mortality worldwide, sensitive and accurate viral diagnostics are crucial for guiding clinical care. In this study, we investigated clinical respiratory samples from a single location in Kenya that had failed to return a diagnosis with the local PCR diagnostic panel. We used randomly primed (unbiased) metagenomic sequencing to increase the viral detection potential. The objectives of this study were twofold. Firstly, we were interested in the number of missed virus diagnoses in respiratory cases. Secondly, we sought respiratory infections in Kilifi whose agents were not included in the routine screening panel. Here we have demonstrated the utility of direct deep sequencing of clinical respiratory samples to identify virus genomes circulating in a resource-limited country. When applying this simple strategy of random-primed viral NGS in respiratory samples testing negative in local diagnostic panels, viral sequences were identified and the approach revealed several categories of missed diagnostics. Category 1 The virus was in the diagnostic panel but locally circulating strains differed at diagnostic primer/probe sites. This is remedied by updating the diagnostic panel with locally appropriate primers, as being done recently ${ }^{2}$. Category 2 The virus was detected but below the cut-off for a positive diagnosis. The current cut-off for positive diagnosis is $\mathrm{PCR} C \mathrm{t}<35.0$; yet the assays did not show Ct-values of negative diagnoses, hence limiting the interpretation of findings here. Further studies 


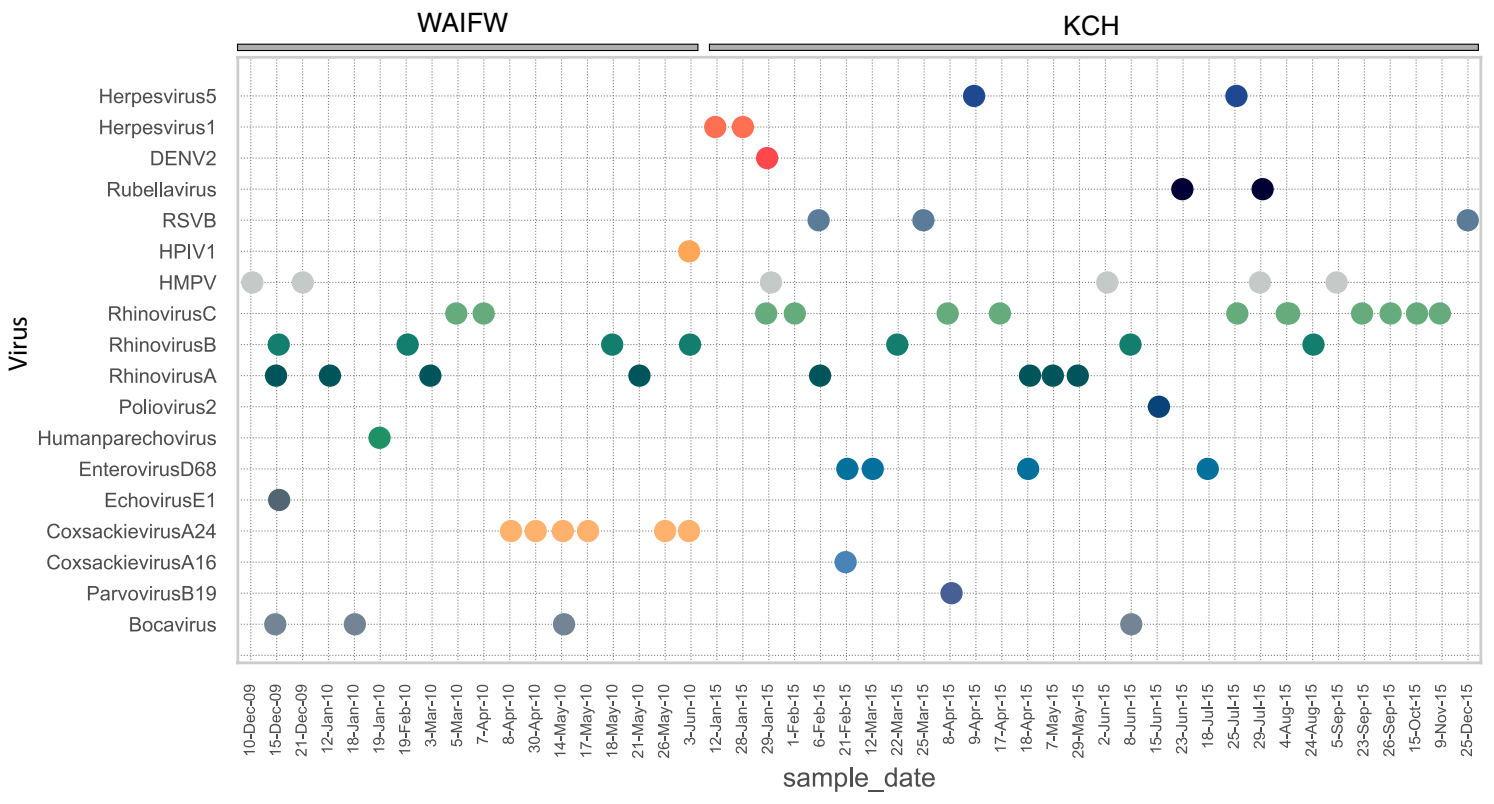

Figure 5. The timeline of viruses identified in the household cohort and $\mathrm{KCH}$ pneumonia patients. The viruses detected in the study (by row) were plotted against the date from which samples were collected (by column). Each virus was presented as a different colour. All the positive samples from household cohort were from 10 December 2009 to 3 June 2010, while positive samples from KCH patients were from 12 January 2015 to 25 December 2015.

are needed to determine assay sensitivities to update positive/negative cutoffs. Category 3 The virus was not in the diagnostic test panel. Due to practical constraints, it is not feasible to include all potential pathogens in a diagnostic panel, however findings from NGS analyses may support the decision to modify diagnostic panels accordingly. A limitation of this NGS approach is the threshold below which a virus does not yield identifiable sequences. Although $30.5 \%$ of the samples returned a viral diagnosis, $69.5 \%$ failed to yield a classifiable viral sequences. We expect that future improvements in NGS methods will increase the fraction of new diagnoses allowed by these methods.

The unexpected/not-tested viruses (i.e. rubella virus, poliovirus, EV-D68), providing additional possible causative agents explaining the symptoms and could help in the local guidelines and policy for disease management and practice. Common viruses were found that had been missed by diagnostics (e.g. HMPV, HRV and RSV). In many cases these detected virus sequences showed nucleotide changes in the primer and/or probe target sites that may account for the missed detection. Although rhinoviruses are the most common virus diagnosis in the Kilifi setting, they were frequently missed by the diagnostics ${ }^{7,18}$. Co-infections with multiple viruses were also detected which may also account for disease severity. Finally, the methods yielded complete or nearly complete genome sequences for respiratory viruses circulating in Kilifi providing a valuable sequence resource for improving local PCR diagnostic assays.

Although viral NGS would be expensive to apply for all diagnostics, the data from this study can inform an optimum pace of applying agnostic viral NGS to improve local diagnostics. The concept of the idea is illustrated in Supplementary Fig. S3. We expect declining diagnostic sensitivity over time (Supplementary Fig. S3, red dashed lines) due to virus evolution with altered primer target sequences, movement into the region of undetectable variants or viruses not on the diagnostic panel. Each round of NGS would result in a revised diagnostic panel, adjusted for local sequence variation and new viruses ("reset sensitivity", Supplementary Fig. S3).

The data and analyses presented here provide a description of circulating respiratory viruses from two cohorts (severe pneumonia from hospital admissions and mild respiratory infections from a household cohort) from one region in coastal Kenya. Albeit small sample size and from a single location, the study included patients with a wide range of symptoms ranging from runny nose, sneezing to severe and very severe pneumonia, with all case types failing to yield a diagnosis for an aetiological pathogen. The study setting and methodologies, i.e. the combination of agnostic viral NGS on samples that had also been subjected to the local viral diagnostic panel, are the strength of the study. This combination allowed us to make important conclusions about the number and type of viruses missed by the local viral diagnostic panel and therefore provides useful information for improving local viral diagnostics.

\section{Materials and methods}

Study location and sample selection. Samples were randomly chosen from an acute respiratory disease surveillance at Kilifi County Hospital (KCH; a primary care and referral hospital) ${ }^{5}$ and a household cohort investigating Who-Acquires-Infection-From-Whom (WAIFW) ${ }^{9}$ from Kilifi, coastal Kenya. The hospital surveillance enrolled paediatric patients presenting with severe or very severe pneumonia based on clinical symptoms as 
previously defined ${ }^{4,5}$, and included children aged 1 day to 59 months and excluded babies with neonatal tetanus. For the KCH surveillance, naso- and oropharyngeal swab (NP/OP) samples were collected as soon as possible after hospital admission as previously described ${ }^{19}$. Ninety-five samples which had tested negative for the 15 respiratory viruses (see "Standard diagnostic panel" section) were randomly selected from January to December 2015 and represent the severe spectrum of respiratory infections in this study.

For the household cohort, members of households in a rural coastal in Kilifi were enrolled and an NP swab was collected from all members irrespective of respiratory symptoms at regular twice-weekly visits from December 2009 to June $2010^{9}$. Ninety-five samples from household members with symptoms of upper respiratory tract infection, which had tested negative for viral pathogens diagnosis, were chosen and represent the mild spectrum of respiratory infections in this study.

Standard diagnostic panel. All samples were screened by multiplex real-time PCR for 15 respiratory virus targets ${ }^{9,18,20}$ : respiratory syncytial virus (RSVA and B), influenza virus A, B, and C, human rhinovirus (HRV), human coronavirus (OC43, NL63 and 229E), adenovirus (AdV), human parainfluenza virus (HPIV1-4), and human metapneumovirus (HMPV). Primers, probes and target genes used for the PCR assays are summarised in Supplementary Table S1. Samples were considered positive when PCR cycle threshold (Ct) was $<35.0$ for any of the 15 virus targets. For KCH samples, RSV antigen was determined using a direct ImmunoFluorescent Antibody Test (IFAT) (Light Diagnostic ${ }^{\mathrm{mm}}$ RSV DFA kit, Chemicon, Millipore Corporation, USA) ${ }^{5}$. The multiplex real-time PCR with or without the IFAT assay is referred to as "the standard diagnostic panel" throughout the manuscript.

Sample preparation and agnostic deep sequencing. Total nucleic acid extraction and dsDNA conversion were performed as previously described ${ }^{21}$. Briefly, the method includes centrifugation and DNase treatment to remove free non-encapsidated DNA, reverse transcription with non-ribosomal random hexamers avoiding rRNA targets followed by sequencing on Illumina HiSeq 2500, generating 2-3 million 250 nt pairedend reads/sample.

De novo assembly and identification of total viral genomes. Quality controlled reads (median Phred $>35$, read length $\geq 175 \mathrm{nt}$, using QUASR ${ }^{22}$ ) were de novo assembled (SPAdes v.3.10 ${ }^{23}$ ). Virus contigs were identified with UBLAST ${ }^{24}$ using virus family protein databases. Final quality control of genomes included checking open reading frames (ORFs), and comparison with reference sequences retrieved from GenBank. Stringent criteria for calling a sample positive for required greater than or equal to $1000 \mathrm{nt}$ (or largest viral segment for segmented viruses).

Phylogenetic construction. Global reference sequences were retrieved from GenBank, coding regions from reference and assembled genomes were extracted, and aligned using MAFFT ${ }^{25}$ and manually checked in AliView $^{26}$. Maximum-likelihood phylogenetic trees were constructed in RAxML ${ }^{27}$ under the Generalised Time Reversible model (GTR) with 100 pseudoreplicates. Bootstrap values of $\geq 70 \%$ were considered statistically significant and were shown for major nodes. Tree was visualised in FigTree v.1.4.3 (http://tree.bio.ed.ac.uk/softw are/figtree). The tree was mid-point rooted for clarity and horizontal branch lengths were drawn to the scale of nucleotide substitutions per site.

Ethical approval. The study was approved by the Kenyan Medical Research Institute Scientific and Ethics Review Unit (KEMRI-SERU) and the Coventry Research Ethics Committee (United Kingdom), and all methods were performed in accordance with the relevant guidelines and regulations by KEMRI-SERU and the Coventry Research Ethics Committee. Written informed consent was obtained for all eligible participants before sample collection. For children ( $<18$ years), informed consent was given by parents or guardians.

\section{Data availability}

Viral genome sequences from this study were deposited in GenBank (accession numbers MK989713-MK989765, Supplementary Table S2).

Received: 3 June 2021; Accepted: 2 December 2021

Published online: 07 January 2022

\section{References}

1. McAllister, D. A. et al. Global, regional, and national estimates of pneumonia morbidity and mortality in children younger than 5 years between 2000 and 2015: A systematic analysis. Lancet Glob. Health 7, e47-e57 (2018).

2. Kamau, E. et al. Recent sequence variation in probe binding site affected detection of respiratory syncytial virus group B by realtime RT-PCR. J. Clin. Virol. 88, 21-25 (2017).

3. Klungthong, C. et al. The impact of primer and probe-template mismatches on the sensitivity of pandemic influenza A/H1N1/2009 virus detection by real-time RT-PCR. J. Clin. Virol. 48, 91-95 (2010).

4. Berkley, J. A. et al. Viral etiology of severe pneumonia among Kenyan young infants and children. J. Am. Med. Assoc. 303, 2051-2057 (2010).

5. Nokes, D. J. et al. Incidence and severity of respiratory syncytial virus pneumonia in rural Kenyan children identified through hospital surveillance. Clin. Infect. Dis. 49, 1341-1349 (2009).

6. Onyango, C. O. et al. Molecular epidemiology of human rhinovirus infections in Kilifi, Coastal Kenya. J. Med. Virol. 84, 823-831 (2012). 
7. Nyiro, J. U. et al. Surveillance of respiratory viruses in the outpatient setting in rural coastal Kenya: Baseline epidemiological observations. Wellcome Open Res. 3, 89 (2018).

8. Agoti, C. et al. Transmission patterns and evolution of RSV in a community outbreak identified by genomic analysis. Virus Evol. 3, 1-12 (2017).

9. Munywoki, P. K. et al. The source of respiratory syncytial virus infection in infants: A household cohort study in rural Kenya. J. Infect. Dis. 209, 1685-1692 (2014).

10. Tan, S. K. et al. Metagenomic sequencing of stool samples in Bangladeshi infants: Virome association with poliovirus shedding after oral poliovirus vaccination. Sci. Rep. 10, 15392 (2020).

11. Yinda, C. K. et al. Gut virome analysis of cameroonians reveals high diversity of enteric viruses, including potential interspecies transmitted viruses. mSphere 4, e00585 (2019).

12. Owor, B. E. et al. Human metapneumovirus epidemiological and evolutionary patterns in Coastal Kenya, 2007-11. BMC Infect. Dis. 16, 1-12 (2016).

13. Kamau, E. et al. An intensive, active surveillance reveals continuous invasion and high diversity of rhinovirus in households. J. Infect. Dis. 219, 1049-1057 (2019).

14. Cobbin, J. C. A. et al. A complex mosaic of enteroviruses shapes community-acquired hand, foot and mouth disease transmission and evolution within a single hospital. Virus Evol. 4, 1-11 (2018).

15. Altan, E. et al. Enteric virome of Ethiopian children participating in a clean water intervention trial. PLoS ONE 13, 1-20 (2018).

16. Enfissi, A. et al. Coxsackievirus A24 variant associated with acute haemorrhagic conjunctivitis cases, French Guiana, 2017. Intervirology 60, 271-275 (2018).

17. World Health Organization. Kenya Rolls Out Massive Measles-Rubella and Tetanus Campaign (WHO, 2016).

18. Munywoki, P. K. et al. Continuous invasion by respiratory viruses observed in rural households during a respiratory syncytial virus seasonal outbreak in coastal Kenya. Clin. Infect. Dis. 67, 1559-1567 (2018).

19. Hammitt, L. L. et al. A preliminary study of pneumonia etiology among hospitalized children in Kenya. Clin. Infect. Dis. 54, S190-S199 (2012).

20. Gunson, R. N., Collins, T. C. \& Carman, W. F. Real-time RT-PCR detection of 12 respiratory viral infections in four triplex reactions. J. Clin. Virol. 33, 341-344 (2005).

21. Phan, M. V. et al. Unbiased whole-genome deep sequencing of human and porcine stool samples reveals circulation of multiple groups of rotaviruses and a putative zoonotic infection. Virus Evol. 2, 58875 (2016).

22. Watson, S. J. et al. Viral population analysis and minority-variant detection using short read next-generation sequencing. Philos. Trans. R. Soc. Lond. B Biol. Sci. 368, 20120205 (2013).

23. Bankevich, A. et al. SPAdes: A new genome assembly algorithm and its applications to single-cell sequencing. J. Comput. Biol. J. Comput. Mol. Cell Biol. 19, 455-477 (2019).

24. Edgar, R. C. Search and clustering orders of magnitude faster than BLAST. Bioinformatics 26, 2460-2461 (2010).

25. Katoh, K. \& Standley, D. M. MAFFT multiple sequence alignment software version 7: Improvements in performance and usability. Mol. Biol. Evol. 30, 772-780 (2013).

26. Larsson, A. AliView: A fast and lightweight alignment viewer and editor for large datasets. Bioinformatics 30, 3276-3278 (2014).

27. Stamatakis, A. RAxML-VI-HPC: Maximum likelihood-based phylogenetic analyses with thousands of taxa and mixed models. Bioinform. Oxf. Engl. 22, 2688-2690 (2006).

\section{Acknowledgements}

We are grateful to all household participants in the household cohort and the enrolled patients and their families who consented to the $\mathrm{KCH}$ hospital surveillance that made this study possible. We thank all field workers for their efforts to visit the households and collect the samples. We thank the Illumina B team at the Wellcome Trust Sanger Institute (Hinxton, Cambridge, UK) for their help in library preparation and deep sequencing. We also acknowledge Andrew Egesa for his assistance on the literature background information. We additionally thank Dr Sarah Atkinson (KEMRI, Kilifi, Kenya) for her stimulating comments and discussion on rubella infection. The study is published with the permission of the Director of KEMRI.

\section{Author contributions}

Study design and conceptualization: M.V.T.P., M.C., P.K., C.N.A. and D.J.N.; Metadata: G.P.O., M.V.T.P. and D.J.N.; Sample logistics: M.V.T.P., P.K., P.K.M., C.N.A., G.P.O., M.N. and D.J.N.; Sequence data analysis: M.V.T.P. and M.C.; Genbank submission: M.C.; First draft preparation: M.V.T.P. and M.C.; Writing-review and editing: M.V.T.P., C.N.A., D.J.N. and M.C.; Final manuscript approval: all co-authors.

\section{Funding}

This study was funded by the Wellcome Trust (Grant Numbers 102975, 090853).

\section{Competing interests}

The authors declare no competing interests.

\section{Additional information}

Supplementary Information The online version contains supplementary material available at https://doi.org/ 10.1038/s41598-021-03987-1.

Correspondence and requests for materials should be addressed to M.V.T.P. or D.J.N.

Reprints and permissions information is available at www.nature.com/reprints.

Publisher's note Springer Nature remains neutral with regard to jurisdictional claims in published maps and institutional affiliations. 
(c) (i) Open Access This article is licensed under a Creative Commons Attribution 4.0 International cc) License, which permits use, sharing, adaptation, distribution and reproduction in any medium or format, as long as you give appropriate credit to the original author(s) and the source, provide a link to the Creative Commons licence, and indicate if changes were made. The images or other third party material in this article are included in the article's Creative Commons licence, unless indicated otherwise in a credit line to the material. If material is not included in the article's Creative Commons licence and your intended use is not permitted by statutory regulation or exceeds the permitted use, you will need to obtain permission directly from the copyright holder. To view a copy of this licence, visit http://creativecommons.org/licenses/by/4.0/.

(C) The Author(s) 2022 